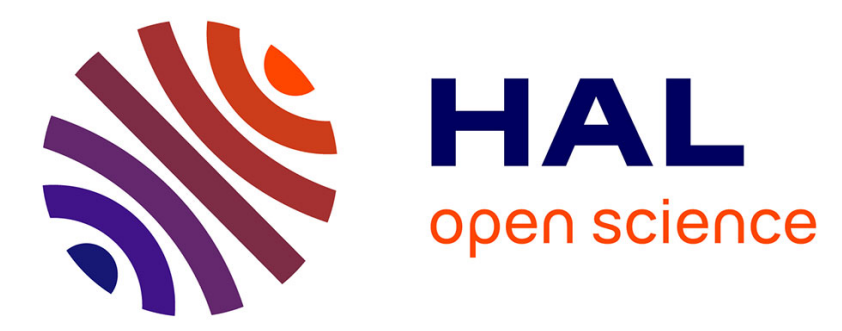

\title{
A systematic review evaluating the methodological aspects of meta-analyses of genetic association studies in cancer research
}

\author{
Stefania Boccia, Emma Feo, Paola Gallì, Francesco Gianfagna, Rosarita
} Amore, Gualtiero Ricciardi

\section{To cite this version:}

Stefania Boccia, Emma Feo, Paola Gallì, Francesco Gianfagna, Rosarita Amore, et al.. A systematic review evaluating the methodological aspects of meta-analyses of genetic association studies in cancer research. European Journal of Epidemiology, 2010, 25 (11), pp.765-775. 10.1007/s10654-010-9503-z . hal-00621303

\section{HAL Id: hal-00621303 https://hal.science/hal-00621303}

Submitted on 10 Sep 2011

HAL is a multi-disciplinary open access archive for the deposit and dissemination of scientific research documents, whether they are published or not. The documents may come from teaching and research institutions in France or abroad, or from public or private research centers.
L'archive ouverte pluridisciplinaire HAL, est destinée au dépôt et à la diffusion de documents scientifiques de niveau recherche, publiés ou non, émanant des établissements d'enseignement et de recherche français ou étrangers, des laboratoires publics ou privés. 
Title: A systematic review evaluating the methodological aspects of meta-analyses of genetic association studies in cancer research

Stefania Boccia*, Emma De Feo, Paola Gallì, Francesco Gianfagna, Rosarita Amore, Gualtiero Ricciardi

Affiliation: Institute of Hygiene, Università Cattolica del Sacro Cuore, Rome, Italy

* Corresponding author 


\begin{abstract}
Background. Meta-analyses and Individual Patient Data (IPD) meta-analyses of genetic association studies are a powerful tool to summarize the scientific evidences, however their application present considerable potential and several pitfalls.
\end{abstract}

Methods. We reviewed systematically all published meta-analyses and IPD meta-analyses of genetic association studies in the field of cancer research, searching for relevant studies on the Medline, Embase, and HuGE Reviews Archive databases until January 2009. The association between selected predictors of methodological quality and the year of publication was also evaluated.

Results. 144 meta-analyses involving 299 gene-disease associations, and 25 IPD meta-analyses on 83 gene-disease were included. Overall quality of the reports showed a substantial improvement over time, as authors have become more inclusive of primary papers published in all languages since 2005 ( $p$-value=0.087), and statistical heterogeneity and publication bias were evaluated more systematically. Only $35.4 \%$ of the meta-analyses, however, adopted a comprehensive bibliographic search strategy to identify the primary reports, $63.9 \%$ did not specify the language of the included studies, 39.8\% did not test for Hardy-Weinberg Equilibrium (HWE), and $62.2 \%$ and $75.9 \%$ of the meta-analyses and IPD meta-analyses, respectively, did not declare the scientific rationale for the genetic model chosen. Additionally, the HWE assessment showed a substantial decreasing trend over time ( $p$-value $=0.031)$ while publication bias was more often evaluated when statistical heterogeneity was actually present ( $p$-value $=0.007)$.

Conclusion. Although we showed a general methodological improvement over time, guidelines on conducting and reporting meta-analyses of genetic association studies are needed to enhance their methodological quality. 


\section{Introduction}

Continuous advances in genotyping technologies and the inclusion of DNA collection in observational studies have resulted in an increasing number of genetic association studies in the field of cancer research. The CDC database of the published literature ${ }^{1}$ reveals that the annual number of reports on genetic association involving cancer has increased impressively in the past ten years, and over 9,300 papers have been published so far. (http://hugenavigator.net/HuGENavigator/searchSummary.do).

Alongside with such a remarkable growth of evidence has come an increasing need to collate the results through the meta-analytical procedure. This allows both to increase the power to detect a true genetic association, as most of the studies in the field are largely underpowered, and to investigate the potential sources of heterogeneity between studies ${ }^{2}$.

Currently, systematic reviews and meta-analyses are a common approach to summarise genedisease associations: almost 500 meta-analyses and Individual Patient Data (IPD) meta-analyses have been published so far, with 360 and 86 meta-analyses and IPD meta-analysis (http://hugenavigator.net/HuGENavigator/searchSummary.do), respectively, in the field of cancer research. ${ }^{1}$

The growth of meta-analysis, however, has not gone unchallenged. The credibility of a metaanalysis depends on the reliability of the included data, on the adoption of an appropriate methodology, and on the correct interpretation and reporting of the evidence. While several efforts have been made to share best practices, tools and methods for design, analysis and appraisal of genetic association studies, ${ }^{3-5}$ and more recently to strengthen their report, ${ }^{6}$ few coordinated initiatives have been produced to standardise the methods for conducting and reporting metaanalyses of genetic association studies, such as the HuGENet handbook ${ }^{7}$ and the recently published paper by Sagoo et al. ${ }^{8}$ A systematic review of 37 meta-analyses of genetic association studies published before 2000 and identified through Medline database, showed their poor methodological quality, ${ }^{9}$ with only $80 \%$ mentioning the bibliographic search strategy, and $19 \%$ checking for 
publication bias. A more recent review from Minelli et al. ${ }^{10}$ evaluated the quality of 120 randomly selected meta-analyses of genetic association studies published between 2005-2007 and identified through HuGE Reviews Archive (http://www.cdc.gov/genomics/hugenet/reviews:arch.htm). After identifying some quality indicators, authors conclude that the overall quality of conducting and reporting genetic meta-analyses improved after 2000 and provided some specific recommendations. The paper, however, did exclude IPD meta-analyses, and reviewed genetic meta-analyses published over a short time period in all the medical fields with no specific disease group addressed.

Our paper aims to be more comprehensive as systematically reviews the methodological aspects and the potential for bias of all the meta-analyses and IPD meta-analyses of genetic association studies published to date in the field of cancer research. We also aim to examine the trend of some methodological issues related with the quality of the study over time.

\section{METHODS}

\section{Eligibiliy criteria}

Published meta-analyses and IPD meta-analyses evaluating the effect of common gene variants on cancer were considered. Only papers written in English were considered for further evaluation. A common gene variant was defined as having a population frequency of at least $1 \% .^{11}$ Studies concerning the effect of gene variants on (disease free) survival from cancer, or cancer precursors were excluded. Studies addressing the relationship between genes expression and cancer risk or prognosis were also excluded, as well as narrative reviews and systematic reviews not accompanied by a meta-analysis.

\section{Information Sources}

Studies were identified through electronic databases search and scanning the reference lists of the eligible articles. No restrictions on language were applied. This search was carried out on Medline (1966- $31^{\text {st }}$ January 2009), and Embase (1980-31 ${ }^{\text {st }}$ January 2009). Databases search was conducted 
by FG using the following search terms: ((meta-analysis) or (pooled analysis)) and (polymorphism) and (cancer)). We also searched for additional studies in the HuGE Reviews Archive (http://www.cdc.gov/genomics/hugenet/reviews:arch.htm).

\section{Study Selection}

EDF and PG performed eligibility assessment independently and following blinded and standardised procedures. Articles were screened by reading their titles and abstracts, and in some cases the entire publications. Disagreement between reviewers was solved by consensus.

\section{Data collection process}

We developed a data extraction sheet, pilot tested on 4 studies randomly selected from those meeting the inclusion criteria, and redefined accordingly. Disagreements were solved by discussion between the two reviewing authors; if no agreement could be reached, a third reviewing author (SB) would settle the difference. The information extracted from each included report consisted in: 1) year of publication, 2) country of the corresponding author; 3) declaration of any kind of financial support declared in the appropriate section of the paper; 4) bibliographic search methodology used; 5) restrictions applied to the language of the studies included in the report, if any; 6) method for quality assessment (quality score, only brief comment in the discussion, or none); 7) tumour site; 8) name of the genetic variant investigated; 9) statistical data pooling or qualitative report; 10) statistical significance ( set at $p$-value $=0.05$ ) of the summary effect estimate [Odds Ratio $(\mathrm{OR}) /$ Risk ratio (RR)]; 11) statistical heterogeneity evaluation ( $Q$-Cochrane test, $I^{2}$, or both); 12) publication bias checking (funnel plot, Begg and Egger tests); 13) Hardy Weinberg Equilibrium (HWE) test of the primary studies included (evaluated, not evaluated, or not applicable if the number of heterozygotes was not provided by the analytical technique used); 14) genetic model used (single genetic model, multiple models, per-allele analysis, or a combination of the previous), and 15) its scientific rationale (fully understandable, not reported, or authors declaring to adopt one previously 
used in published reports). The data source (Genetic Susceptibility to Environmental Carcinogens, consortia or data collection from the individual researchers), and the type of included studies (published or unpublished) were recorded only for the IPD meta-analyses.

The impact factors (IF) of the journals in which the studies were published are relative to the Journal Citation Reports 2007

(http://thomsonreuters.com/products_services/science/science_products/a-

z/journal_citation_reports). Genetic variants were grouped according to the gene family.

\section{Statistical analysis}

Since our a priori hypothesis was that the methodological quality of meta-analyses improved over time, we evaluated the association between selected predictors of quality (bibliographic search strategy, language restriction, assessment of heterogeneity, publication bias and HWE evaluation) and the year of publication (until 2004 versus 2005 onwards, as a sharp increase of publication was detected across these two years). We also tested the association between the publication bias assessment (when meta-analyses included at least ten primary studies) as predictor of the quality of the meta-analysis itself, and bibliographic search strategy, overall OR/RR, presence of heterogeneity, and impact factor of the journal. $\chi^{2}$ test and Fisher's exact test were used, when appropriate. Statistical significance was defined as a two-sided $p$-value $<0.05$ for all analyses, which were carried out using the STATA software package v.10.0 (Stata Corporation, College 162 Station, Texas).

\section{RESULTS}

\section{Study selection}

A total of 144 meta-analyses (Supplementary data) involving 299 gene-diseases associations, and 25 IPD meta-analyses on 83 gene-diseases were included in the review. Figure 1 details the flow of information through the different phases of the systematic review. The search through the Medline, Embase and HuGE Reviews Archive databases provided a total of 300 citations on meta-analyses 
and 90 on IPD meta-analyses after duplicates removal. 127 meta-analyses were discharged because after reviewing the titles and abstracts it appeared clear that these studies did not meet the inclusion criteria. 1 additional paper was discarded because full texts was not available (Supplementary data). By examining the full texts of the remaining 173 citations in closer detail, 28 meta-analyses were later excluded. As for the IPD meta-analyses, 50 out of 90 were discharged after reading titles and abstracts, as clearly they did not meet inclusion criteria. The full texts of the remaining 40 citations were examined in closer detail, and 15 were excluded. Reasons for excluding both the 29 metaanalyses and the 15 IPD meta-analyses previously mentioned were because studies concerned: (i) chronic non-cancer diseases, or (ii) low frequency gene variant $(<1 \%)$, or (iii) overall survival from cancer as endpoint, and (iv) micro-array data.

\section{Characteristics of the meta-analyses}

The oldest meta-analysis identified was published in $1993^{12}$; the number of published meta-analysis increased sharply after 2004 (Table 1). The vast majority of the corresponding authors were Europeans (43.7\%), followed by Asians (27.1\%). More than half of the meta-analyses were financially supported, and $52.8 \%$ were published on scientific journals with an IF ranging from 3.1 to 5.0 (Table 1). The bibliographic search strategy mostly consisted in Medline and hand search as the sole approach to retrieve the published articles (39.6\% of the meta-analyses), while only $35.4 \%$ adopted a more comprehensive strategy, including at least the Medline database, an additional electronic database and references hand search. Surprisingly, $8.3 \%$ of the studies did not report the bibliographic search strategy methods adopted (Table 1). Most of the meta-analyses (63.9\%) did not specify if any exclusion criteria was applied based on the language of the primary reports. Quality assessment using a published score system was performed in $6.2 \%$ of the studies (Table 1).

Table 2 reviews the 299 genetic associations investigated in the 144 meta-analyses included, and details the methodology adopted to carry out the meta-analysis. The vast majority of the polymorphisms considered involved glutathione S-transferases gene families (19.1\%), and 
methyltetrahydrofolate gene (11.0\%). Lung was the most common cancer site investigated (18.4\%), followed by breast (15.0\%), and colorectum (12.0\%). 62.9\% of the meta-analyses reported the absence of a statistically significant association between gene polymorphisms and cancer risk (Table 2). The random effect model was the most commonly used (61.6\%), even though $55.2 \%$ of the studies assessing the statistical heterogeneity actually reported the absence of any heterogeneity at $p$-value $\leq 0.05$. About $31 \%$ of the meta-analyses used the $I^{2}$ when measuring the statistical heterogeneity, while $67.7 \%$ of the studies among those including at least 10 primary reports evaluated the publication bias properly and performed at least one statistical test (Begg or Egger tests). $84.2 \%$ of the meta-analyses including less than 10 primary studies, however, did check for publication bias. Subgroup analysis was carried out in $76.9 \%$ of the studies, while HWE was not evaluated in $39.8 \%$ of the reports (Table 2). Lastly, even though $58.7 \%$ adopted one single genetic model to perform the meta-analysis while $34.9 \%$ used multiple models, the scientific rationale for the choice of the model was not declared by $62.2 \%$ of the meta-analysts.

\section{Characteristics of the IPD meta-analyses}

The oldest IPD meta-analysis identified was published in $2000,{ }^{13}$ with more than $50 \%$ published from January 2008 to January 2009 (Table 3). The vast majority of the corresponding authors were Europeans (56.0\%), and $88 \%$ of the studies were financially supported. The Genetic Susceptibility to Environmental Carcinogens (GSEC) project was the most common initiative aimed at the collection of individual datasets, followed by ad hoc established consortia (36.0\%). $44.0 \%$ of the IPD meta-analyses included both published and unpublished primary studies (Table 3). Overall, the 25 IPD meta-analyses retrieved evaluated the effect of 83 genetic associations, the vast majority of which involving glutathione S-transferases gene families (14.5\%), followed by X-ray repair crosscomplementing family (10.8\%). Again, lung was the most studied cancer site (32.5\%), followed by breast $(30.1 \%)$ and ovary (15.7\%). $30.1 \%$ of the IPD meta-analyses did not specify the method used to pool the data, and most of the studies (68.7\%) were unable to detect a statistically significant 
association between the gene polymorphisms and cancer outcomes (Table 3). Absence of heterogeneity at $p$-value $\leq 0.05$ was reported in $61.5 \%$ of the IPD meta-analyses, and HWE of the primary studies included was evaluated in $73.5 \%$ of the IPD meta-analyses (Table 3). 55.4\% adopted multiple genetic models, and $75.9 \%$ did not specify the scientific rationale behind the model choice.

Characteristics of the meta-analyses according to the year of publication and relationship between publication bias evaluation and selected items

As reported in Table 4, the quality of the bibliographic search strategy did not improve significantly over time ( $p$-value $=0.484)$. Even if we were unable to detect a statistically significant association between publication year and language restriction $(p$-value $=0.087), 22.5 \%$ of the meta-analyses published after 2005 did not apply restrictions on the language of the primary studies included, compared to $9.1 \%$ of those published before. Heterogeneity assessment increased over time, $(p$ value $=0.014)$, as evaluation of publication bias for meta-analyses including at least 10 primary studies $(p$-value $\leq 0.001)$. As for publication bias assessment in meta-analyses including less than 10 primary studies, figures decreased over time, but not significantly ( $p=0.161$ ) (Table 4$)$. HWE evaluation, however, showed an opposite trend, with a significantly reduced number of studies checking for deviations from the equilibrium $(p$-value $=0.031)$.

No relationship was detected between publication bias evaluation and the bibliographic search strategy $(p$-value $=0.725)$ and with the statistical significance of the pooled estimate ( $p$-value $=0.670$ ). The evaluation of publication bias related inversely to the presence of heterogeneity, with the vast majority of meta-analyses checking for publication bias mostly in the presence of a statistical heterogeneity among the data $(p$-value $=0.007)$. Lastly, publication bias checking was strongly associated with the IF of the publishing journals: meta-analyses evaluating publication bias were more likely $(82.8 \%)$ to be published in journals with an IF $\geq 3.0(p$-value $=0.003)($ Table 4$)$. 


\section{DISCUSSION}

Meta-analyses are the most cited study design in health sciences ${ }^{14}$ and are widely accepted as the highest level of evidence in medicine. The strengths and limitations of systematic reviews are well established for clinical trials, largely through the efforts of the Cochrane Collaboration. ${ }^{15}$ In the field of genetic association studies, systematic reviews and meta-analyses in the past few years have become a common approach. ${ }^{3}$ Their value is in their capability of integrating evidence on the presence and magnitude of gene-disease associations, and they should be designed as rigorous research studies. ${ }^{16}$ The recently published Preferred Reporting Items for Systematic reviews and Meta-Analyses (PRISMA) statement, which updates the QUORUM checklist ${ }^{17}$, attempted to improve standards for reporting systematic reviews and meta-analyses in all the field of the epidemiological research, with a particular emphasis on randomized trials. ${ }^{18}$ Even though most of the items included in the PRISMA checklist also apply to the field of genetic medicine, detailed consensus guidelines on how to conduct and report a meta-analysis of genetic association studies is currently lacking.

This is the first systematic review of all published meta-analyses and IPD meta-analyses of genetic association studies in the field of cancer research published so far. As expected, published genetic associations reflect the epidemiology of cancer, as the most common cancer sites for which metaanalyses were published were lung, breast and colorectum. ${ }^{19}$ We showed a substantial improvement in methodological quality over time, even though several key items are still liable to further improvements. We showed that only $35.4 \%$ of the meta-analyses adopted a comprehensive bibliographic search strategy to identify the eligible primary reports; that $63.9 \%$ did not specify the language of the included studies; that only $21.6 \%$ measured the size of the statistical heterogeneity with the $I^{2}$ test; that $39.8 \%$ did not test for HWE; and that $62.2 \%$ and $75.9 \%$ of the meta-analyses and IPD meta-analyses, respectively, did not declare the scientific rationale for their choice of a specific genetic model. 
A comprehensive bibliographic search strategy is a crucial step to avoid selection bias in a metaanalysis. The National Library of Medicine's Medline database is one of the most comprehensive sources of health care information in the world. Like any database, however, its coverage is not complete and varies according to the specific field of health care. ${ }^{18}$ The issue of a comprehensive search strategy to retrieve the greatest number of available studies for a meta-analyses has been addressed for clinical trials ${ }^{20}$ and observational studies ${ }^{21}$ both showing that at least Medline, another electronic database plus hand searching should be used to provide a thorough summary of existing published literature. Even though the issue has not been explored in the field of genetic association studies, from our results we argue that a large amount of the meta-analyses published so far, at least in the field of cancer research, might be biased because of the incomplete retrieval of the studies, and this should be carefully considered. A different issue pertains the inclusion of unpublished reports, and the impact of this approach on the overall effect estimate. Retrieving unpublished data retrospectively might be unfeasible, and in this sense the consortia represent a defence against publication and selection reporting biases..$^{22} 44 \%$ of the IPD meta-analyses in the present review did include both published and unpublished reports. Even though a recent study showed that metaanalyses of published data and consortium analyses generate identical results, ${ }^{23}$ we believe that consortium analyses offer higher opportunity to perform rare subgroup analyses, or gene-gene and gene-environment interaction analyses if compared with the meta-analyses. As such, their creation should be encouraged.

We showed that only $19.4 \%$ of the meta-analyses clearly declared to include primary studies of all languages. An empirical evaluation of the Chinese literature published in 2005 showed that language bias affects the field of human genome epidemiology, and this may reasonably not be limited to the Chinese literature. ${ }^{24}$ Therefore, in order to avoid introducing additional selection bias, authors should be encouraged to include and translate non-English articles in their meta-analyses. 
We showed that, oppositely to the bibliographic search strategy that did not show major improvements over time, since 2005 authors have become more inclusive of primary papers published in all languages.

So far, 86 tools for grading the quality of epidemiological studies have been published. ${ }^{25}$ These, however, have major limitations ${ }^{26}$ both because of the subjective component in the application of the scale, and because of the actual difficulty to produce a valid assessment of quality. Our systematic review showed that $6.2 \%$ of the meta-analyses used a published quality score system to grade the primary studies included. Only $26.4 \%$, however, discussed the methodological weakness and strength of the individual reports, which should be one of the primary issue to be explored in a meta-analysis, regardless of the quality scale adopted .

As far as methodological aspects of data pooling are concerned, $61.6 \%$ of the meta-analyses used a random effect model. Heterogeneity has a direct bearing on deciding whether and how to pool results. Random effect model is appropriate both in absence and in presence of statistical heterogeneity, even though the fixed-effect model might be more appropriate when studies are homogeneous. ${ }^{27}$ Some authors, however, stress the importance of dissecting the source of heterogeneity rather than pooling data; in our systematic review, 76.9\% did perform subgroup analyses. This positive aspect is even more prominent in the IPD meta-analyses (96.4\% performed subgroup analyses). Additionally, $79.7 \%$ of the reviewed meta-analyses evaluating heterogeneity were published after 2004, confirming an improvement over the years. This trend has been reported also from Minelli et al. ${ }^{10}$

Higgins et al. ${ }^{28}$ in 2003 proposed the use of the $I^{2}$ test as a measurement of the size of heterogeneity between studies. $I^{2}$ test defines as the percentages of total variation in study estimates explained by heterogeneity rather than sampling error, and it has a higher power and does not depend on the 
number of primary studies included compared to the Q-statistics ${ }^{28}$. By keeping in mind that 114 meta-analyses have been published in the period 2004-2008, our review shows the $I^{2}$ was not used until 2005 when it was adopted by just two meta-analyses, while it has been used by 18 (16\%) meta-analyses published in 2008. These data are a bit lower than those reported by Minelli et al. ${ }^{10}$ showing that $31 \%$ of 120 randomly selected meta-analyses published between 2005 and 2007 adopted the $I^{2}$ test for heterogeneity evaluation, nevertheless they show an increasing use of the $I^{2}$ test in the heterogeneity assessment.

Methods for pooling data in IPD meta-analysis depend on the available data. Usually, a two-step approach is applied. Firstly, the risk estimates and variance for each study are calculated and adjusted for the same covariates across the studies included, using a multivariable model. Secondly, a combined estimate is obtained as a weighted average of the individual estimates, using a random or fixed effect model usually based on the result of the heterogeneity evaluation. In our systematic review, $30.1 \%$ of the IPD meta-analyses did not specify how data were actually pooled, while $38.5 \%$ used a random effect model, so there is room for an improvement in reporting the actual statistical methodology adopted for the data pooling.

Publication bias refers to the possibility that negative results do not have the same chance of being published as positive results. Graphic methods to detect publication bias based on the asymmetric distribution of the effect sizes plotted versus some measure of precision have been suggested (e.g., funnel plot), however their exclusive use has been discouraged. ${ }^{29}$ Our systematic review showed that publication bias was evaluated by means of a formal statistical test (Egger or Begg tests) in $67.7 \%$ of the meta-analyses among those including at least 10 primary reports. We also reported that publication bias was properly evaluated in $75.6 \%$ of the studies published since 2005. A systematic review of 37 meta-analyses of genetic association studies published before 2000 showed that no study used a formal statistical test to detect publication bias, ${ }^{9}$ whereas the review from 
Minelli et al. ${ }^{10}$ reported that $58 \%$ of the meta-analyses published between 2005 and 2007 did, thus confirming this positive trend. The evaluation of publication bias using a formal statistical test might be considered an indicator of the quality of the meta-analyses, thus we investigated its relationship with the bibliographic search strategy and the impact factor of the journal, finding a positive relation between them. The presence of publication bias, however, was more often evaluated when statistical heterogeneity was present, while we expected the opposite situation. Even though testing for publication bias is always recommended for meta-analyses including at least 10 studies, its use could be redundant in presence of a substantial statistical heterogeneity and leading to false-positive claims, as the presence of heterogeneity itself is already indicative of the absence of publication bias. ${ }^{30}$

We hypothesized that studies that did not evaluate publication bias properly were more likely to show significant effect estimate at $p$-value $\leq 0.05$, however we glad not to observe it.

A large amount of meta-analyses including less than 10 primary studies, however, did check for publication bias, although in these cases the use was inappropriate because of the very low statistical power of the Begg and Egger tests and the limited added value of the funnel plot. ${ }^{30}$ Our results show a decreasing proportion, however, of this attitude over the years.

The evaluation of HWE should always be performed individually in all the studies included, as deviation from HWE might indicate the presence of genotyping errors, population stratification or selection bias among the control populations. ${ }^{31}$ From our systematic review, $39.8 \%$ out of 144 meta-analyses did not test for HWE. Oppositely, $73.5 \%$ of the IPD meta-analyses tested for HWE, and that is suggestive of their general higher quality compared with meta-analyses. The systematic review of 37 meta-analyses of genetic association studies published before 2000 from Attia et al. ${ }^{9}$ showed that only $24 \%$ of them tested for HWE, whereas the review from Minelli et al. ${ }^{10}$ reported that $41 \%$ of the meta-analyses published between 2005 and 2007 actually tested for HWE. Surprisingly, our data highlights that there is an inverse relationship between the HWE testing and the year of publication, showing a substantially decreasing trend over time. 
A critical step in any meta-analysis of genetic association studies is the adoption of a specific genetic model. From our systematic review, $58.7 \%$ of the meta-analyses and $44.6 \%$ of the IPD meta-analyses adopted a single genetic model, and 3.7\% compared the allele frequencies, which is based on the assumption of an additive genetic model. As suggested by Attia et al. ${ }^{9}$, the choice of model should express genotype risk in a meaningful way according to biological background. As the a priori knowledge to support the choice of the correct genetic model is often lacking, some researchers perform multiple pairwise comparison, which require an adjustment for multiple testing to avoid false positive findings. From our systematic review more than $35 \%$ of the meta-analyses fitted multiple models actually, even though no one of them adjusted for multiple comparisons.

By comparing the results of our study with the systematic review from Attia et al. ${ }^{9}$ and the review of Minelli et al. ${ }^{10}$ we observed an increase in meta-analyses adopting a single genetic model (33 and $27 \%$, respectively). Since clear knowledge to choose the true genetic model derived from biological studies might have increased over time, we speculate researchers might have used a model previously adopted by influential papers on the same genetic variant thus potentially explaining the difference proportion between our systematic review and the previous ones.

From our systematic review we found that the scientific rationale was clearly explained in $30.2 \%$ and $21.7 \%$ of the meta-analyses and IPD meta-analyses, respectively. Additionally, among the meta-analyses and IPD meta-analyses adopting a single genetic model, the percentages of those declaring the rationale behind was $43.4 \%$ and $43.2 \%$, respectively.

In conclusion, meta-analysis is a powerful methodological tool that requires expertise and strict adherence to certain methodological steps. The present systematic review shows that the general quality of the meta-analyses of genetic association studies in the field of cancer research has improved over time, even though it is generally low when compared to IPD meta-analyses and still poor for some aspects discussed in the present review. Since some of the reviewed IPD metaanalyses are structured as HuGE reviews, following the indication of the HuGE review handbook ${ }^{7}$, 
we argue that this has positively influenced the way these studies have been conducted and their results have been reported. Few differences emerged between our review and the one from Minelli et al. ${ }^{10}$, which however randomly included meta-analyses published between 2005 and 2007 addressing all field of medical research in genetic epidemiology. These differences might be attributable to the peculiar focus of our review that specifically addressed cancer genetics epidemiology by retrieving all meta-analyses and IPD meta-analyses published so far. In spite of this, we argue that there is a need for specific consensus guidelines to be developed by expert network of investigators, that should take into account both the HuGE review handbook and the PRISMA statement on how to conduct and report a meta-analysis of genetic association studies. 


\section{Acknowledgment}

We thank Benedetto Simone for the English style revision of the final draft of the manuscript and Jessica Rowell and Alessia Melegaro for data collection. 


\section{REFERENCES}

1 Lin BK, Clyne M, Walsh $\mathrm{M}$ et al. Tracking the epidemiology of human genes in the literature: the HuGE Published Literature database. Am J Epidemiol 2006;164:1-4.

2 Ioannidis JP. Genetic associations: false or true?. Trends Mol Med 2003;9:135-8.

3 Ioannidis JP, Gwinn M, Little J et al. A road map for efficient and reliable human genome epidemiology. Nat Genet 2006;38:3-5.

4 Hattersley AT, McCarthy MI. What makes a good genetic association study?. Lancet 2005;366:1315-23.

Ioannidis JP, Boffetta P, Little $\mathrm{J}$ et al. Assessment of cumulative evidence on genetic associations: interim guidelines. Int J Epidemiol 2008;37:120-32.

6 Little J, Higgins JP, Ioannidis JP et al. Strengthening the reporting of genetic association studies (STREGA): an extension of the strobe statement. Ann Intern Med 2009;150:206215.

7 Higgins JP, Little J, Ioannidis JP et al. Turning the pump handle: evolving methods for integrating the evidence on gene-disease association. Am J Epidemiol 2007;166:863-6.

8 Sagoo GS, Little J, Higgins JP. Systematic Reviews of Genetic Association Studies. Plos med 2009;6:e1000028. 
9 Attia J, Thakkinstian A, D'Este C. Meta-analyses of molecular association studies: Methodologic lessons for genetic epidemiology. J Clin Epidemiol 2003;56:297-303.

Minelli C, Thompson JR, Abrams KR, Thakkinstian A, Attia J. The quality of metaanalyses of genetic association studies: a review with recommendations. Am J Epidemiol 2009;170:1333-43.

Suh Y, Vijg J. SNP discovery in associating genetic variation with human disease phenotypes. Mutat Res 2005;573:41-53.

Krontiris TG, Devlin B, Karp DD, Robert NJ, Risch N. An association between the risk of cancer and mutations in the HRAS1 minisatellite locus. N Engl J Med 1993;329:517-523.

Marcus PM, Hayes RB, Vineis $\mathrm{P}$ et al. Cigarette smoking, $\mathrm{N}$-acetyltransferase 2 acetylation status, and bladder cancer risk: a case-series meta-analysis of a gene-environment interaction. Cancer Epidemiol Biomarkers Prev 2000;9:461-7.

Patsopoulos NA, Analatos AA, Ioannidis JP. Relative citation impact of various study designs in the health sciences. JAMA 2005;293:2362-2366.

Chalmers I. The Cochrane collaboration: preparing, maintaining, and disseminating systematic reviews of the effects of health care. Ann N Y Acad Sci 1993;703:156-63. 2006;3:9-13. 
17 Moher D, Cook DJ, Eastwood S, Olkin I, Rennie D, et al. Improving the quality of reports of meta-analyses of randomised controlled trials: The QUOROM statement. Quality of Reporting of Meta-analyses. Lancet 1999;354:1896e900

Liberati A, Altman DG, Tetzlaff $\mathrm{J}$ et al. The PRISMA statement for reporting systematic reviews and meta-analyses of studies that evaluate health care interventions: explanation and elaboration. J Clin Epidemiol 2009;62:e1-34.

19 Boyle P, Ferlay J. Cancer incidence and mortality in Europe, 2004. Ann Oncol 2005;16:4818.

Hopewell S, Clarke M, Lefebvre C, Scherer R. Handsearching versus electronic searching to identify reports of randomized trials. Cochrane Database Syst Rev 2007;2:MR000001.

21 Lemeshow AR, Blum RE, Berlin JA et al. Searching one or two databases was insufficient for meta-analysis of observational studies. J Clin Epidemiol. 2005; 58(9):867-73

Seminara D, Khoury MJ, O'Brien TR et al. The emergence of networks in human genome epidemiology: challenges and opportunities. Epidemiology 2007;18:1-8.

23 Janssens AC, González-Zuloeta Ladd AM, López-Léon S et al. An empirical comparison of meta-analyses of published gene-disease associations versus consortium analyses. Genet Med 2009;11:153-62. 
Pan Z, Trikalinos TA, Kavvoura FK, Lau J, Ioannidis JP. Local literature bias in genetic epidemiology: an empirical evaluation of the Chinese literature. PloS Med 2005;2:e334.

Sanderson S, Tatt ID, Higgins JP. Tools for assessing quality and susceptibility to bias in observational studies in epidemiology: a systematic review and annotated bibliography. Int $J$ Epidemiol 2007;36:666-76.

Herbison P, Hay-Smith J, Gillespie WJ. Adjustment of meta-analyses on the basis of quality scores should be abandoned. J Clin Epidemiol 2006;59:1249-56.

Egger M, Davey Smith G, Altman DG. Systematic Rewiers in Health Care. Meta - analysis in context. London, Uk:BMJ Publishing Group, 2001.

Higgins JP, Thompson SG, Deeks JJ, Altman DG. Measuring inconsistency in metaanalyses. BMJ 2003;327:557-60.

Lau J, Ioannidis JP, Terrin N, Schmid CH, Olkin I. The case of the misleading funnel plot. BMJ 2006;333: 597-600.

Ioannidis JP, Trikalinos TA. The appropriateness of asymmetry tests for publication bias in meta-analyses: a large survey. CMAJ 2007 Apr 10;176:1091-6.

31 Attia J, Ioannidis JP, Thakkinstian A et al. How to use an article about genetic association: A: Background concepts. JAMA 2009;301:74-81. 
Figure 1. Flowchart depicting literature search and selection.

Potentially relevant articles identified through PubMed database

Meta-analyses $=220$

IPD $^{\text {a }}$ meta-analyses $=139$
Potentially relevant articles identified through EMBASE database

Meta-analyses $=294$

IPD meta-analyses $=213$
Potentially relevant articles identified through $\mathrm{HuGE}$ Reviews Archive Database

Meta-analyses $=176$

IPD meta-analyses $=78$

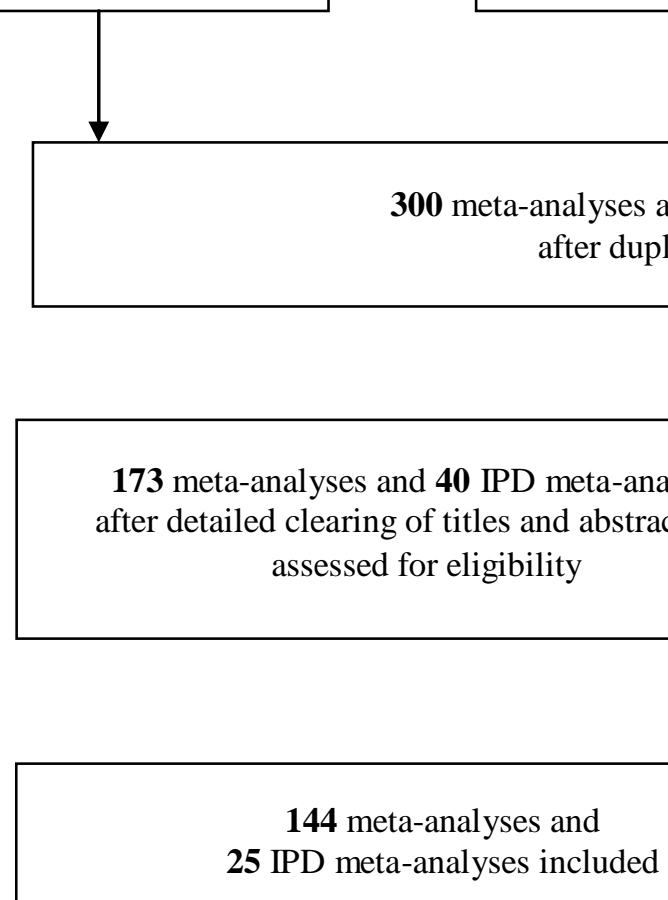

${ }^{\mathrm{a}} \mathrm{IPD}=$ Individual patient data

${ }^{\mathrm{b}}=$ Not meeting inclusion criteria (See results section for details)

${ }^{\mathrm{c}}=$ full text not available

\section{Table 1. Description of the meta-analyses included $(\mathrm{N}=144)$}

Excluded:

28 meta-analyses $^{\mathbf{b}}$

15 IPD meta-analyses ${ }^{\mathbf{b}}$

1 meta-analysis ${ }^{\mathbf{c}}$

\section{Year of publication}

January 2009

Number $(\%)$ 
Before 2000

$6(4.2)$

\section{Continent of the corresponding author}

Europe

$63(43.7)$

Asia

$39(27.1)$

North America

$37(25.7)$

Australia

$3(2.1)$

South America

$2(1.4)$

Impact factor

No Impact factor

$4(2.8)$

$\leq 1.5$

$5(3.5)$

$1.6-3.0$

$27(18.7)$

$3.1-5.0$

$76(52.8)$

$5.1-10.0$

$23(16.0)$

$>10.0$

$9(6.2)$

Financial support

Declared

$78(54.2)$

Not declared

$66(45.8)$

\section{Bibliographic search}

Medline and hand search

$57(39.6)$

Medline, EMBASE and hand search

$28(19.4)$

Medline, electronic databases different from

$16(11.1)$

EMBASE and hand search

Medline only

$15(10.4)$

Unspecified methods

$12(8.3)$

Medline, EMBASE, other electronic databases and hand search

Medline, EMBASE, other electronic databases

Medline and electronic databases different from

$5(3.5)$

EMBASE

Medline and EMBASE

$1(0.7)$

Other methods

$1(0.7)$

\section{Language of included studies}

Unspecified

$92(63.9)$

All languages

$28(19.4)$

English only

$19(13.2)$

English and Chinese

$3(2.1)$

English, Spanish and other language

$1(0.7)$

English and Spanish

$1(0.7)$

Quality assesment

Not assessed

$97(67.4)$

Brief comment in discussion section

$38(26.4)$

Quality score used

$9(6.2)$ 
Table 2. Description of the meta-analyses included according to the genetic associations evaluated $(\mathbf{N}=\mathbf{2 9 9})$

\begin{tabular}{lc}
\hline & Number (\%) \\
\hline Gene families/Genes & \\
Glutathione S-transferases $(G S T$ s $)$ & $57(19.1)$ \\
Methyltetrahydrofolate $(M T H F R)$ & $33(11.0)$ \\
Cytochrome P450 variants $(C Y P)$ & $28(9.4)$ \\
X-ray repair cross-complementing family $(X R C C)$ & $25(8.4)$ \\
p53 & $18(6.0)$ \\
Xeroderma pigmentosum genes $(X P)$ & $18(6.0)$ \\
N-acetyltransferase 2 genes $(N A T-2)$ & $14(4.7)$ \\
Interleukins $(I L s)$ & $12(4.0)$ \\
Growth factor genes variants $(G F)$ & $11(3.7)$ \\
Vitamin D receptor gene $(V D R)$ & $11(3.7)$ \\
Cyclooxygenases gene variants $(C O X)$ & $8(2.7)$ \\
Excision repair cross complementing $(E R C C)$ & $5(1.7)$ \\
Tumor Necrosis Factor variants $(T N F)$ & $5(1.7)$ \\
8q24 gene variants & $4(1.3)$ \\
Estrogen receptor alpha gene variants & $2(0.7)$ \\
Others & $48(16.0)$ \\
\hline Cancer site &
\end{tabular}

\section{Cancer site}

Lung

$55(18.4)$

Breast

Colorectum

$36(12.0)$

Stomach

$34(11.4)$

Prostate

$32(10.7)$

Head and neck

$17(5.7)$

Bladder

Hematopoietic malignancies

$11(3.7)$

Esophagus

$7(2.3)$

Brain glioma

$4(1.3)$

Uterine cervix

Cancer overall

$36(12.0)$

\section{Overall OR/RR ${ }^{\mathrm{a}}$}

Not statistically significant ${ }^{b}$

$188(62.9)$

Statistically significant

$$
>1.0
$$

Not calculated

$$
<1.0
$$

$7(2.3)$

\section{Quantitative pooling data method}

Random effects model

Fixed effects model

Both models

\section{Statistical heterogeneity evaluation}

Assessed

Present at a $p$-value $<0.05$

Present (level of significance not reported ) 
Statistical test for heterogeneity evaluation

Q-test

$94(66.2)$

$Q$-test and $I^{2}$

$35(24.7)$

$9(6.3)$

Unspecified

$4(2.8)$

Publication bias check for meta-analyses including $\mathbf{1 0}$

studies

Funnel plot and Begg or Egger tests

$116(58.6)$

Not performed

Begg or Egger tests only

$18(9.1)$

Funnel plot only

$9(4.5)$

Publication bias check for meta-analyses including $<10$

studies

Performed (any method)

$85(84.2)$

Not performed

$16(15.8)$

Subgroup analysis

Performed

$230(76.9)$

Not performed

$69(23.1)$

HWE test of the primary studies included

Yes

$150(51.0)$

No

$117(39.8)$

Not applicable $27(9.2)$

\section{Genetic model used}

Single genetic model

$175(58.7)$

Multiple models tested

$104(34.9)$

Allelic frequencies

$11(3.7)$

Unspecified

Allelic frequencies and multiple models

Allelic frequencies and single model

$2(0.7)$

\section{Scientific rationale for the genetic model used clearly stated}

Not reported

Fully understandable

Previously used

$22(7.6)$

${ }^{\mathrm{a}} \mathrm{OR}=$ Odds Ratio, RR = Risk Ratio

${ }^{\mathrm{b}}$ Statistical significance set at $p$-value $<0.05$.

Table 3. Description of the individual patient data meta-analyses of genetic polymorphisms 
Year of publication

January 2009

2008

2007

2006

2005

2004

2003

2002

2001

2000

\section{Number (\%)}

$6(24.0)$

7 (28.0)

$1(4.0)$

$2(8.0)$

$1(4.0)$

$1(4.0)$

$3(12.0)$

$2(8.0)$

$0(0.0)$

$1(4.0)$

\section{Impact factor}

$<3.0$

$3.0-5.0$

$3(12.0)$

$15(60.0)$

7 (28.0)

\section{Continent of the corresponding author}

Europe

$14(56.0)$

North America

$10(40.0)$

Asia $1(4.0)$

\section{Financial support}

Declared

$22(88.0)$

Not declared

$3(12.0)$

\section{Data source}

Genetic Susceptibility to Environmental

$12(48.0)$

Carcinogens

Consortia

$9(36.0)$

Data collection from single authors

\section{Included studies}

Published and unpublished

$4(16.0)$

Only published studies

$11(44.0)$

7 (28.0)

Only unpublished studies

Description of the individual data meta-analyses according to the genetic associations

$(\mathbf{N}=\mathbf{8 3})$

\section{Gene families/Genes}

Glutathione S-transferases (GSTs)

$12(14.5)$

$\mathrm{X}$-ray repair cross-complementing family

$9(10.8)$

$(X R C C)$

Cytochrome $\mathrm{P} 450$ variants $(C Y P)$

$9(10.8)$

Progesterone receptor gene variants $(P G R)$

p53

$3(3.6)$

Excision repair cross complementing (ERCC) 3 (3.6)

Growth factor genes variants $(G F)$

Alcohol dehydrogenase $(A D H)$

Vitamin D receptor gene (VDR)

Methyltetrahydrofolate (MTHFR)

Microsomal Epoxide hydrolase $(E P H X)$

$\mathrm{N}$-acetyltransferase 2 genes (NAT-2)

Other 


\section{Cancer site}

Lung

27 (32.5)

Breast

$25(30.1)$

Ovary

$13(15.7)$

Head and neck

$9(10.9)$

Bladder

$2(2.4)$

Endometrium

$2(2.4)$

Stomach

$2(2.4)$

Esophagus

$1(1.2)$

Colorectum

$1(1.2)$

Cancer overall

$1(1.2)$

\section{Pooling data method}

Random effects model

$32(38.5)$

Random and fixed effects models

Unspecified

$25(30.1)$

Fixed effects model

$3(3.6)$

Unconditional logistic regression

$2(2.4)$

Conditional logistic regression

\section{Overall OR/RR ${ }^{\mathrm{a}}$}

Not statistically significant ${ }^{\mathrm{b}}$

$2(2.4)$

Statistically significant

$57(68.7)$

$26(31.3)$

$20(76.9)$

$>1.0$

$6(23.1)$

\section{Heterogeneity assessment}

Assessed

$76(91.6)$

Absent

$51(61.5)$

Present at a $p$-value $<0.05$

$19(22.9)$

Not reported

$6(7.2)$

Not assessed

$7(8.4)$

\section{Subgroup analysis}

Performed

$80(96.4)$

Not performed

3 (3.6)

HWE test of the primary studies included

Yes

$61(73.5)$

No

Not applicable

$10(12.0)$

\section{Genetic model used}

Single genetic model

37 (44.6)

Multiple models tested

$46(55.4)$

\section{Scientific rationale for the genetic model}

\section{used clearly stated}

Not reported

Fully understandable

Previously used

${ }^{\mathrm{a}} \mathrm{OR}=$ Odds Ratio, RR = Risk Ratio

${ }^{\mathrm{b}}$ Statistical significance set at $p$-value $<0.05$. 


\begin{tabular}{|c|c|c|c|}
\hline \multicolumn{4}{|c|}{$\begin{array}{l}\text { Table 4. Relationship between the year of publication and the publicatio } \\
\text { with selected items from the } 144 \text { meta-analyses } \\
\text { Year of publication }\end{array}$} \\
\hline & until 2004 & 2005 onwards & $p$-value \\
\hline \multicolumn{4}{|c|}{ Bibliographic search strategy ${ }^{a}$} \\
\hline Comprehensive & $10(30.3)$ & $41(36.9)$ & 0.484 \\
\hline Not comprehensive & $23(69.7)$ & $70(63.1)$ & \\
\hline \multicolumn{4}{|l|}{ Language of included studies } \\
\hline All languages & $3(9.1)$ & $25(22.5)$ & 0.087 \\
\hline Some restriction applied & $30(90.9)$ & $86(77.5)$ & \\
\hline \multicolumn{4}{|l|}{ Heterogeneity assessment } \\
\hline Evaluated & $58(90.6)$ & $228(97.4)$ & 0.014 \\
\hline Not evaluated & $6(9.4)$ & $6(2.6)$ & \\
\hline \multicolumn{4}{|c|}{$\begin{array}{l}\text { Publication bias evaluation for } \\
\text { meta-analyses } \\
\text { including less than } 10 \text { primary } \\
\text { studies }\end{array}$} \\
\hline Performed $^{\mathrm{b}}$ & $25(92.6)$ & $60(81.1)$ & 0.161 \\
\hline Not performed & $2(7.4)$ & $14(18.9)$ & \\
\hline \multicolumn{4}{|c|}{$\begin{array}{l}\text { Publication bias evaluation for meta-analyses } \\
\text { including } \geq \mathbf{1 0} \text { primary studies }{ }^{c}\end{array}$} \\
\hline Performed & $13(34.2)$ & $121(75.6)$ & 0.000 \\
\hline Not performed ${ }^{\mathrm{d}}$ & $25(65.8)$ & $39(24.4)$ & \\
\hline \multicolumn{4}{|l|}{ HWE test of included studies } \\
\hline Yes & $46(71.9)$ & $131(57.0)$ & 0.031 \\
\hline \multirow[t]{3}{*}{ No } & $18(28.1)$ & $99(43.0)$ & \\
\hline & \multicolumn{3}{|c|}{$\begin{array}{c}\text { Publication bias evaluation for meta-analyses } \\
\text { including } \geq 10 \text { primary studies }\end{array}$} \\
\hline & Performed $^{\mathrm{c}}$ & Not performed & \\
\hline \multicolumn{4}{|c|}{ Bibliographic search strategy ${ }^{a}$} \\
\hline Comprehensive & $25(41.7)$ & $9(37.5)$ & 0.725 \\
\hline Not comprehensive & $35(58.3)$ & $15(62.5)$ & \\
\hline \multicolumn{4}{|l|}{ Overall OR/RR $\mathbf{R}^{\mathrm{e}}$} \\
\hline Statistically significant & $52(40.3)$ & $27(43.6)$ & 0.670 \\
\hline Not statistically significant $^{\mathrm{f}}$ & $77(59.7)$ & $35(56.4)$ & \\
\hline \multicolumn{4}{|c|}{$\begin{array}{l}\text { Heterogeneity presence at } p \text {-value } \\
<0.05^{\mathrm{g}}\end{array}$} \\
\hline No & $52(40.0)$ & $35(61.4)$ & 0.007 \\
\hline Yes & $78(60.0)$ & $22(38.6)$ & \\
\hline \multicolumn{4}{|l|}{ Impact factor } \\
\hline$<3.0$ & $22(16.9)$ & $17(27.4)$ & 0.003 \\
\hline $3.0-5.0$ & $70(53.6)$ & $40(64.5)$ & \\
\hline$>5.0$ & $38(29.2)$ & $5(8.1)$ & \\
\hline
\end{tabular}


${ }^{a}$ Bibliographic search was considered comprehensive if including at least Medline, another electronic database plus references hand search

${ }^{\mathrm{b}}$ By any method including funnel plot and/or Egger or Begg test

${ }^{\mathrm{c}}$ By Egger and/or Begg test

${ }^{\mathrm{d}}$ Also including the sole use of funnel plot

${ }^{\mathrm{e}} \mathrm{OR}=$ Odds Ratio, $\mathrm{RR}=$ Risk Ratio

${ }^{\mathrm{f}}$ Statistical significance set at $p$-value $<0.05$

${ }^{\mathrm{g}}$ Heterogeneity evaluated for 286 gene associations. 